\title{
Bar optimal design with the use of out-form
}

\author{
Vadim Yu. Alpatov ${ }^{1, *}$, Andrei A. Sakharov ${ }^{1}$, Oleg Yu. Veremeenko ${ }^{1}$ and Alexey A. Lukin ${ }^{1}$ \\ ${ }^{1}$ Samara State Technical University Institute of Architecture and Civil Engineering, \\ Molodogvardeyskaya st. 194, 443001 Samara, Russia
}

\begin{abstract}
The paper describes steel girders optimal design. Steel girders optimal design is based on the strength theory introduced by M. Huber, R. Mises, H. Hencky and on the theory of mirror functions. With the help of out-form volume we can either calculate the optimal form for a girder with variable cross-section or the optimal height for a girder of uniform crosssection. The paper introduces girders calculations according to the authors' methodology. For use this methodology effect of steel saving is about 10$20 \%$ compared with a girder or a beam with uniform cross-section. An example of determining optimal shape of a compressed-bend girder the paper describes.
\end{abstract}

\section{Introduction}

There are several well-known methods of building structures optimal design $[1,2,5,6,7$, 8] that can be applied while designing building structures cross-beams or girders. In this paper, the authors present an example of determining optimal shape of a compressed-bend. The paper introduces girders calculations according to the authors' methodology.

\section{Bar optimal design with the use of out-form}

According to theory [3], strength in this case is provided on condition that:

$$
(1+\mu) \cdot \frac{R_{y}^{2}}{3} \cdot E
$$

For a plane stress problem, direct stress in $i$ point of the bar can be determined from the formula:

$$
\sigma_{i c}=\frac{N_{i c}}{F_{i c}}+\frac{M_{i c}}{W_{i c}}
$$

Specific potential energy of the shape change in $i$ point of the bar is

$$
\frac{1+\mu}{3 \cdot E} \cdot\left(\frac{N_{i c}}{F_{i c}}+\frac{M_{i c}}{W_{i c}}\right)^{2}+\frac{1}{2 \cdot G} \cdot \frac{Q_{i c}^{2} \cdot S_{i c}^{2}}{I_{i c}^{2} \cdot \sigma_{i c}^{2}} \leq[u]_{\Phi}
$$

\footnotetext{
*Corresponding author: avu75@mail.ru
} 
As a result of transformations we can introduce a new equation from the strength condition. This equation is mathematically determined as non-linear modular form i bar capacity [4-6]:

$$
V=\frac{l_{i}}{R_{i} \cdot \sqrt{\left[N_{i c}^{2}+2 \cdot N_{i c} \cdot M_{i c} \cdot \alpha_{i c}+M_{i c}^{2} \cdot \alpha_{i c}^{2}+3 \cdot Q_{i c}^{2} \cdot \beta_{i c}^{2}\right]}}
$$

where $\alpha_{i c}=a_{c} / r_{c}^{2}$ and $\beta_{i c}=S_{i c} /\left(r_{c}^{2} \delta_{c}\right)$.

Shape coefficients $\alpha$ і и $\beta \mathrm{i}$ are cross sections accessible characteristics and can be easily derived from gauge material or calculated. With given cross-sectional height, cross-section form coefficients $\alpha$ and $\beta$ are taken as constant.

For a statically indeterminate bar with uniform cross-section the volume function can be presented as it follows:

$$
V_{i}=l_{i} \sqrt{\frac{0.433}{v_{i}[U]_{\phi} E}\left[\begin{array}{l}
\left(N_{i p}+\sum N_{i j} x_{j}\right)^{2}+2 \cdot\left(N_{i p}+\sum N_{i j} x_{j}\right)\left(M_{i p}+\sum M_{i j} x_{j}\right) \alpha_{i}+ \\
+\left(M_{i p}+\sum M_{i j} x_{j}\right)^{2} \alpha_{i}^{2}+3 \cdot\left(Q_{i p}+\sum Q_{i j} x_{j}\right)^{2} \beta_{i}^{2}
\end{array}\right]}
$$

In general terms, Formula 5 can be given as

$$
V_{i}=F_{i} l_{i}=\sqrt{\sum_{j=1}^{n} \sum_{k=1}^{n} A_{i j k} x_{j} x_{k}+2 \sum_{j=1}^{n} B_{i j} x_{j}+C_{i}}
$$

where

$$
\begin{gathered}
A_{i k j}=\frac{l^{2} \cdot 0.433}{v_{i}[U]_{\phi} E}\left[\left(N_{i k} N_{i j}+N_{i k} M_{i j} \alpha_{i}+M_{i k} N_{i j} \alpha_{i}+M_{i k} M_{i j} \alpha_{i}^{2}\right)+3 Q_{i k} Q_{i j} \beta_{i}^{2}\right] \\
B_{i j}=\frac{l^{2} \cdot 0.433}{v_{i}[U]_{\phi} E}\left[\left(N_{i p} N_{i j}+N_{i p} M_{i j} \alpha_{i}+M_{i p} N_{i j} \alpha_{i}+M_{i p} M_{i j} \alpha_{i}^{2}\right)+3 Q_{i p} Q_{i j} \beta_{i}^{2}\right] \\
C_{i}=\frac{l^{2} \cdot 0.433}{v_{i}[U]_{\phi} E}\left[\left(N_{i p}^{2}+2 N_{i p} M_{i p} \alpha_{i}+M_{i p}^{2} \alpha_{i}^{2}\right)+3 Q_{i p}^{2} \beta_{i}^{2}\right]
\end{gathered}
$$

If this statically determinate bar is variable in its cross-section along its length, we can find the optimal shape or height for a uniform cross-section bar while using the form coefficient $\alpha$ and $\beta$. For that we need to find the dependence $\alpha$ and $\beta$ from the crosssectional height h [9]. If we take I-beams (according to All Union State Standard - GOST 8239-89) and compare all the coefficients $\alpha$ and $\beta$ for outer fibers, fibers on flange and wall junction and neutral fibers we'll see that there is an approximate hyperbolic dependence between 3 and $\alpha$ and linear dependence between $h$ and $\beta$ (as Fig. 1 shows).

Paper [7] introduces statistical research proving that the radius of cross-section gyration of a defined height h-const in flexural and compressed flexural elements does not depend on their other characteristics if their construction and calculation constraints are taken into account. The radius of gyration changes only if cross-sectional height is changed. We can see almost the same dependence in respect of the width of (flange) section $b$. 

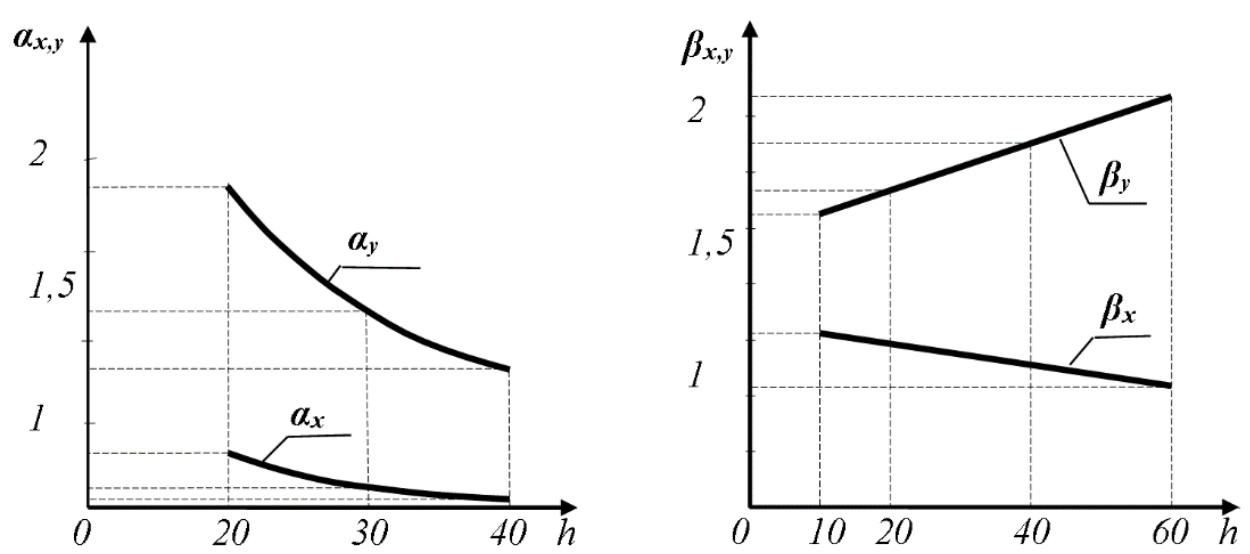

Fig. 1. Dependency diagrams of cross-section form coefficients for I-beams (according to All Union State Standard (GOST 8239-89) $\alpha_{x}, \alpha_{y}, \beta_{x}, \beta_{y}$ from cross-sectional height $\mathrm{h}$.

Tables 1 and 2 show $\alpha, \beta$ coefficients for rolled sections of different types.

Table 1. Cross-section form coefficients $\alpha_{x}, \alpha_{y}$.

\begin{tabular}{|c|c|c|c|c|c|c|c|c|c|}
\hline $\begin{array}{c}\text { Cross- } \\
\text { sectional } \\
\text { height } \\
h(\mathrm{~cm})\end{array}$ & $\begin{array}{l}\text { All Union } \\
\text { State } \\
\text { Standard } \\
\text { (GOST) } \\
\text { 26020-83 }\end{array}$ & $\begin{array}{c}\alpha_{x} \\
(1 / \mathbf{c m})\end{array}$ & $\begin{array}{c}\alpha_{y} \\
(1 / \mathbf{c m})\end{array}$ & $\begin{array}{c}\text { All } \\
\text { Union } \\
\text { State } \\
\text { Standard } \\
\text { (GOST) } \\
\text { 26020-83 }\end{array}$ & $\begin{array}{c}\alpha_{x} \\
(1 / \mathrm{cm})\end{array}$ & $\begin{array}{c}\alpha_{y} \\
(1 / \mathbf{c m})\end{array}$ & $\begin{array}{c}\text { All } \\
\text { Union } \\
\text { State } \\
\text { Standard } \\
\text { (GOST) } \\
\text { 8239-89 }\end{array}$ & $\begin{array}{c}\alpha_{x} \\
(1 / \mathrm{cm})\end{array}$ & $\begin{array}{c}\alpha_{y} \\
(1 / \mathrm{cm})\end{array}$ \\
\hline 10 & - & - & - & - & - & - & I 10 & 0.303 & 1.848 \\
\hline 20 & - & - & - & 20SH1 & 0.146 & 0.576 & I 20 & 0.146 & 1.167 \\
\hline \multirow{2}{*}{30} & 30B1 & 0.098 & 0.748 & $30 \mathrm{SH} 2$ & 0.093 & 0.435 & I 30 & 0.100 & 0.939 \\
\hline & 30B3 & 0.095 & 0.692 & $30 \mathrm{SH} 4$ & 0.092 & 0.426 & - & - & - \\
\hline \multirow{2}{*}{40} & $40 \mathrm{~B} 1$ & 0.076 & 0.674 & $40 \mathrm{SH} 2$ & 0.068 & 0.281 & I 40 & 0.077 & 0.844 \\
\hline & $40 \mathrm{~B} 3$ & 0.072 & 0.611 & 40SH4 & 0.068 & 0.279 & - & - & - \\
\hline \multirow{2}{*}{50} & $50 \mathrm{~B} 1$ & 0.061 & 0.562 & $50 \mathrm{SH} 2$ & 0.056 & 0.299 & I 50 & 0.063 & 0.815 \\
\hline & $50 \mathrm{~B} 3$ & 0.058 & 0.514 & $50 \mathrm{SH} 4$ & 0.057 & 0.306 & - & - & - \\
\hline \multirow{2}{*}{60} & $60 \mathrm{~B} 2$ & 0.049 & 0.447 & $60 \mathrm{SH} 2$ & 0.048 & 0.291 & I 60 & 0.054 & 0.758 \\
\hline & - & - & - & $60 \mathrm{SH} 4$ & 0.051 & 0.317 & - & - & - \\
\hline \multirow{2}{*}{70} & 70B2 & 0.043 & 0.461 & $70 \mathrm{SH} 2$ & 0.043 & 0.307 & - & - & - \\
\hline & 70B4 & 0.041 & 0.400 & 70SH4 & 0.044 & 0.314 & - & - & - \\
\hline 80 & 80B4 & 0.038 & 0.408 & - & - & - & - & - & - \\
\hline \multirow{2}{*}{100} & $100 \mathrm{~B} 2$ & 0.031 & 0.361 & - & - & - & - & - & - \\
\hline & 100B4 & 0.031 & 0.344 & - & - & - & - & - & - \\
\hline
\end{tabular}

Cross-section form coefficients for rolled sections, fabricated sections of simple and complex types, U-sections, etc can be approximate by functions like the following:

$$
\alpha=a_{1}+a_{2} / h \quad \beta=c_{1}+c_{2} \cdot h
$$

For example, the following approximations correspond to wide-flange I-beam gauge:

$$
\begin{aligned}
& \alpha_{x}=0.00318213+2.6285 / h \\
& \beta_{x}=4.302782-0.019985 \cdot h \\
& \alpha_{y}=0.194298+16.4660 / b \\
& \beta_{y}=3.9211329-0.44453 \cdot b
\end{aligned}
$$


Let us put functions (10) into the strength condition (3). Here we get a modular form of the volume as regard to $h$ in $k$ point of the bar.

$$
V_{i c}(h)=F_{i} l_{i}=l_{i} / R \sqrt{\left[N_{i c}^{2}+2 \cdot N_{i c} M_{i c}\left(a_{1}+a_{2} / h\right)_{i c}+M_{i c}^{2}\left(a_{1}+a_{2} / h\right)_{k}^{2}+3 \cdot Q_{i c}^{2}\left(c_{1}+c_{2} h\right)_{i c}^{2}\right]}
$$

For a part of the bar with $d_{z}$ length the elementary capacity can be presented as an outform for $\mathrm{n}$ fiber points:

$$
d V=\left\|V_{1}(h)\right\| V_{2}(h)\left\|V_{3}(h)\right\| \ldots . . V_{n}(h) \| d z
$$

\begin{tabular}{|c|c|c|c|c|c|c|c|c|c|}
\hline $\begin{array}{c}\text { Cross- } \\
\text { section } \\
\text { al } \\
\text { height } \\
h(\mathrm{~cm})\end{array}$ & $\begin{array}{c}\text { All } \\
\text { Union } \\
\text { State } \\
\text { Standar } \\
\text { d } \\
\text { (GOST) } \\
\text { 26020-83 }\end{array}$ & $\begin{array}{c}\beta_{x} \\
(\mathbf{b} / \mathbf{r})\end{array}$ & $\begin{array}{c}\beta_{y} \\
(\mathbf{b} / \mathbf{r})\end{array}$ & $\begin{array}{c}\text { All } \\
\text { Union } \\
\text { State } \\
\text { Standar } \\
\text { d } \\
\text { (GOST) } \\
\text { 26020-83 }\end{array}$ & $\begin{array}{c}\beta_{x} \\
(\mathbf{b} / \mathbf{r})\end{array}$ & $\begin{array}{c}\boldsymbol{\beta}_{y} \\
(\mathbf{b} / \mathbf{r})\end{array}$ & $\begin{array}{c}\text { All } \\
\text { Union } \\
\text { State } \\
\text { Standar } \\
\text { d } \\
\text { (GOST) } \\
\text { 8239-89 }\end{array}$ & $\begin{array}{c}\beta_{x} \\
(\mathbf{b} / \mathbf{r})\end{array}$ & $\begin{array}{c}\boldsymbol{\beta}_{y} \\
(\mathbf{b} / \mathbf{r})\end{array}$ \\
\hline 10 & - & - & - & - & - & - & I 10 & 3.101 & $\begin{array}{c}5.08 \\
0\end{array}$ \\
\hline 20 & - & - & - & $20 \mathrm{SH} 1$ & 3.45 & 2.15 & I 20 & 2.917 & $\begin{array}{c}5.83 \\
4\end{array}$ \\
\hline 30 & $\begin{array}{l}30 \mathrm{~B} 1 \\
30 \mathrm{~B} 3\end{array}$ & $\begin{array}{l}2.724 \\
2.929\end{array}$ & $\begin{array}{l}5.233 \\
4.880\end{array}$ & $\begin{array}{l}30 \mathrm{SH} 2 \\
30 \mathrm{SH} 4\end{array}$ & $\begin{array}{l}3.714 \\
3.656\end{array}$ & $\begin{array}{l}4.349 \\
4.297\end{array}$ & $\begin{array}{c}\text { I } 30 \\
-\end{array}$ & $\begin{array}{c}2.725 \\
-\end{array}$ & $\begin{array}{c}6.29 \\
6 \\
-\end{array}$ \\
\hline 40 & $\begin{array}{l}40 \mathrm{~B} 1 \\
40 \mathrm{~B} 3\end{array}$ & $\begin{array}{l}2.538 \\
2.776\end{array}$ & $\begin{array}{l}5.576 \\
5.063\end{array}$ & $\begin{array}{l}40 \mathrm{SH} 2 \\
40 \mathrm{SH} 4\end{array}$ & $\begin{array}{l}3.934 \\
3.836\end{array}$ & $\begin{array}{l}4.211 \\
4.209\end{array}$ & $\begin{array}{c}\text { I } 40 \\
-\end{array}$ & $\begin{array}{c}2.503 \\
-\end{array}$ & $\begin{array}{c}6.54 \\
2 \\
-\end{array}$ \\
\hline 50 & $\begin{array}{l}50 \mathrm{~B} 1 \\
50 \mathrm{~B} 3\end{array}$ & $\begin{array}{l}2.493 \\
2.748\end{array}$ & $\begin{array}{l}5.615 \\
5.126\end{array}$ & $\begin{array}{l}50 \mathrm{SH} 2 \\
50 \mathrm{SH} 4\end{array}$ & $\begin{array}{l}3.467 \\
3.174\end{array}$ & $\begin{array}{l}4.489 \\
4.642\end{array}$ & $\begin{array}{c}\text { I } 50 \\
-\end{array}$ & $\begin{array}{c}2.321 \\
-\end{array}$ & $\begin{array}{c}6.92 \\
5 \\
-\end{array}$ \\
\hline 60 & $\begin{array}{c}\text { 60B2 } \\
-\end{array}$ & $\begin{array}{c}2.732 \\
-\end{array}$ & $\begin{array}{c}5.145 \\
-\end{array}$ & $\begin{array}{l}60 \mathrm{SH} 2 \\
60 \mathrm{SH} 4\end{array}$ & $\begin{array}{l}3.209 \\
2.686\end{array}$ & $\begin{array}{l}4.662 \\
5.165\end{array}$ & $\begin{array}{c}\text { I } 60 \\
-\end{array}$ & $\begin{array}{c}2.321 \\
-\end{array}$ & $\begin{array}{c}7.20 \\
2 \\
-\end{array}$ \\
\hline 70 & $\begin{array}{l}70 \mathrm{~B} 2 \\
70 \mathrm{~B} 4\end{array}$ & $\begin{array}{l}2.519 \\
2.603\end{array}$ & $\begin{array}{l}5.486 \\
5.233\end{array}$ & $\begin{array}{l}70 \mathrm{SH} 2 \\
70 \mathrm{SH} 4\end{array}$ & $\begin{array}{l}2.925 \\
2.647\end{array}$ & $\begin{array}{l}4.920 \\
5.136\end{array}$ & - & - & $\begin{array}{l}- \\
-\end{array}$ \\
\hline $\begin{array}{c}80 \\
100\end{array}$ & $\begin{array}{c}80 \mathrm{~B} 4 \\
100 \mathrm{~B} 2 \\
100 \mathrm{~B} 4\end{array}$ & $\begin{array}{l}2.434 \\
2.372 \\
2.424\end{array}$ & $\begin{array}{l}5.548 \\
5.772 \\
5.562\end{array}$ & $\begin{array}{l}- \\
- \\
-\end{array}$ & $\begin{array}{l}- \\
- \\
-\end{array}$ & $\begin{array}{l}- \\
- \\
-\end{array}$ & $\begin{array}{l}- \\
- \\
-\end{array}$ & $\begin{array}{l}- \\
- \\
-\end{array}$ & $\begin{array}{l}- \\
- \\
-\end{array}$ \\
\hline
\end{tabular}

Table 2. Cross-section form coefficients $\beta_{x}, \beta_{y}$.

\section{An example of determining optimal shape of a compressed- bend girder}

Let us analyze Figure 2, which shows the process of determining optimal shape of a hingesupported compressed-bend girder of a hydraulic gate $[11,12,13]$ with $12 \mathrm{~m}$ span. Steel design resistance is $\mathrm{R}=240 \mathrm{MPa}=24 \mathrm{kN} / \mathrm{cm}^{2}$. 


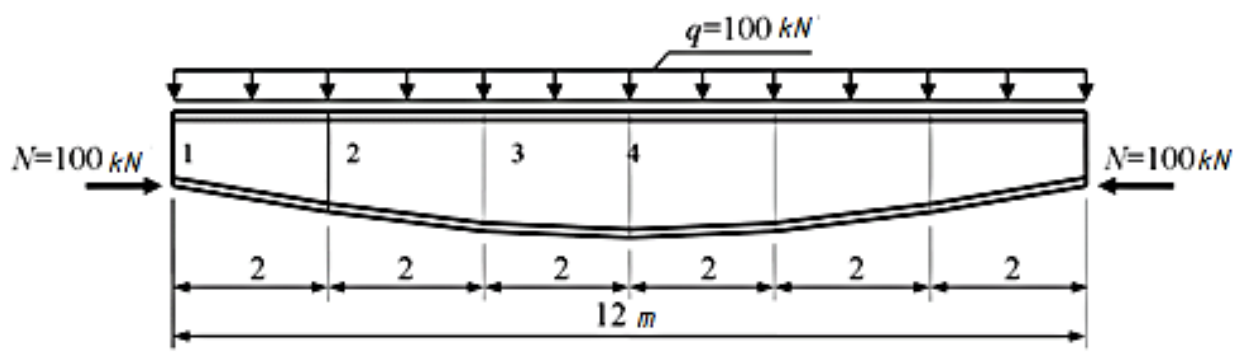

Fig. 2. Compressed-bend girder.

Axial force in a hinge-supported girder is usually applied on the pier level. As Force $\mathrm{N}$ for a beam with uniform cross-section changes the bending moment in the middle of each cross-section, let us determine cross-sectional height in the middle of the span according to strength condition:

$$
W_{x}=M_{\max } / R=\left(q l^{2} / 8\right) / R=\left(50 \cdot 12^{2} / 8\right) / 24=900 / 4=3750 \mathrm{~cm}^{3}
$$

Gauge material for such a moment corresponds to cross-section 60SH2, with $\mathrm{W}_{\mathrm{x}}=4490 \mathrm{~cm}^{3}$.

From the condition of deflection limitation in the middle equal to $1 / 400 \mathrm{~L}=3 \mathrm{~cm}$ we have:

$$
J_{x}=(5 / 48) M_{\max }^{H} l^{2} / 3 E=(5 / 48)\left(50 \cdot 12^{2} / 8\right) /\left(1.2 \cdot 2.05 \cdot 10^{4}\right)=175609 \mathrm{~cm}^{4}
$$

It corresponds to I-beam $70 \mathrm{SH} 2$.

So, from now on we are going to make use of wide-flange I-beams and use the following heights: $\mathrm{H}=(70,60,50,40,30,20)$. According to $\mathrm{H}$ we'll calculate cross-section form coefficients $\alpha_{x 1}, \alpha_{x 2}, \alpha_{x 3}, \beta_{x 1}, \beta_{x 2}, \beta_{x 3}$, and then put them together in Table 3. Here we use gauge material according to GOST 26020-83. Points $1,2,3$ consequently correspond to outer fibers, fibers on flange and wall junction and neutral fibers.

Table 3. Cross-section form coefficients for the line $h$.

\begin{tabular}{lllllll}
\hline $\begin{array}{l}\text { Height } \boldsymbol{h} \\
\text { Coef. }\end{array}$ & $\mathbf{7 0}$ & $\mathbf{6 0}$ & $\mathbf{5 0}$ & $\mathbf{4 0}$ & $\mathbf{3 0}$ & $\mathbf{2 0}$ \\
\hline$\alpha_{x, 1}$ & 0.043 & 0.042 & 0.058 & 0.069 & 0.093 & 0.146 \\
$\beta_{x, 1}$ & 0 & 0 & 0 & 0 & 0 & 0 \\
$\alpha_{x, 2}$ & 0.0395 & 0.038 & 0.054 & 0.065 & 0.096 & 0.1414 \\
$\beta_{x, 2}$ & 1.976 & 1.986 & 2.019 & 2.796 & 2.747 & 3.15 \\
$\alpha_{x, 3}$ & 0 & 0 & 0 & 0 & 0 & 0 \\
$\beta_{x, 3}$ & 2.742 & 2.71 & 2.816 & 3.49 & 3.68 & 3.45 \\
\hline
\end{tabular}

We take into account the symmetry of the girder and analyze cross-sections spaced at intervals of $2 \mathrm{~m}$.

1) Optimal design of a hinged beam with variable cross-section starts with determining its bearing cross-section. In bearing cross-section 1 we have $N_{x}=100 \mathrm{kN}$; $M_{x}=N_{x} \cdot h / 2=100 \cdot h / 2 ; Q_{y}=300 \mathrm{kN}$. Let us take $h=30 \mathrm{~cm}$ cross-sectional height. Then $M_{x}=100 \cdot 15=1500 \mathrm{kN} \cdot \mathrm{cm}$. The area we need is calculated according to equations (4).

$$
F_{11}=1 / 24 \sqrt{\left[100^{2}+2 \cdot 100 \cdot 1500 \cdot 0.093+1500^{2} \cdot 0.093^{2}\right]}=9.98 \mathrm{~cm}^{2}
$$




$$
\begin{aligned}
& F_{12}=1 / 24 \sqrt{\left[100^{2}+2 \cdot 100 \cdot 1500 \cdot 0.096+1500^{2} \cdot 0.096^{2}+3 \cdot 300^{2} \cdot 2.747^{2}\right]}=65.93 \mathrm{~cm}^{2} \\
& F_{13}=1 / 24 \sqrt{\left.100^{2}+2 \cdot 100 \cdot 1500 \cdot 0+1500^{2} \cdot 0+3 \cdot 300^{2} \cdot 3.68^{2}\right]}=79.78 \mathrm{~cm}^{2}
\end{aligned}
$$

We take $h=30 \mathrm{~cm}$ and area $F_{l}=79.78 \mathrm{~cm}^{2}$ along neutral fiber where both shearing force and axial force are quite strong.

2) Let us analyze the cross-section at the distance of $2 m$ from its supporting structure. Let us take $h=40 \mathrm{~cm}$ cross-sectional height. There are forces $N_{x}=100 \mathrm{kN} ; M_{x}=(300 \cdot 2-$ $50 \cdot 2 \cdot 1)-100 \cdot 0.1=400 \mathrm{kN} \cdot \mathrm{m}=40000 \mathrm{kN} \cdot \mathrm{cm} ; Q_{y}=300-100=200 \mathrm{kN}$ in the cross-section. The area we need is

$$
\begin{aligned}
& F_{21}=1 / 24 \sqrt{\left[100^{2}+2 \cdot 100 \cdot 40000 \cdot 0.069+40000^{2} \cdot 0.069^{2}\right]}=117.8 \mathrm{~cm}^{2} \\
& F_{22}=1 / 24 \sqrt{\left[100^{2}+2 \cdot 100 \cdot 40000 \cdot 0.065+40000^{2} \cdot 0.065^{2}+3 \cdot 200^{2} \cdot 2.796^{2}\right]}=119.52 \mathrm{~cm}^{2} \\
& F_{23}=1 / 24 \sqrt{\left[100^{2}+2 \cdot 100 \cdot 40000 \cdot 0+40000^{2} \cdot 0+3 \cdot 200^{2} \cdot 3.68^{2}\right]}=53.3 \mathrm{~cm}^{2}
\end{aligned}
$$

It is clear that in cross-section 2 its area depends on the bending moment and on the tension at flange and wall junction.

Let us take $h=50 \mathrm{~cm}$ cross-sectional height. Let us determine the forces by eccentricity. Here we have $N_{x}=100 \mathrm{kN} ; M_{x}=(300 \cdot 2-50 \cdot 2 \cdot 1)-100 \cdot 0.05=450 \mathrm{kN} \cdot \mathrm{m}=45000 \mathrm{kN} \cdot \mathrm{cm}$; $Q_{y}=300-100=200 \mathrm{kN}$.

$$
\begin{aligned}
& F_{21}=1 / 24 \sqrt{\left[100^{2}+2 \cdot 100 \cdot 45000 \cdot 0.058+45000^{2} \cdot 0.058^{2}\right]}=112.9 \mathrm{~cm}^{2} \\
& F_{22}=1 / 24 \sqrt{\left[100^{2}+2 \cdot 100 \cdot 45000 \cdot 0.054+45000^{2} \cdot 0.054^{2}+3 \cdot 200^{2} \cdot 2.019^{2}\right]}=109.4 \mathrm{~cm}^{2} \\
& F_{23}=1 / 24 \sqrt{\left.100^{2}+2 \cdot 100 \cdot 45000 \cdot 0+45000^{2} \cdot 0+3 \cdot 200^{2} \cdot 2.816^{2}\right]}=40.85 \mathrm{~cm}^{2}
\end{aligned}
$$

We tale $h=50 \mathrm{~cm}$ and the area which is $F_{2}=\operatorname{minmax}=112.9 \mathrm{~cm}^{2}$.

3 ) Let us analyze the cross-section at the distance of $4 \mathrm{~m}$ from its supporting structure. Let us take $h=60 \mathrm{~cm}$ cross-sectional height. There are forces $N_{x}=100 \mathrm{kN} ; M_{x}=(300 \cdot 4-$ $50 \cdot 4 \cdot 2)=800 \mathrm{kN} \cdot \mathrm{m}=80000 \mathrm{kN} \cdot \mathrm{cm} ; Q_{y}=300-200=100 \mathrm{kN}$ in the cross-section. The area we need is

$$
\begin{aligned}
& F_{31}=1 / 24 \sqrt{\left[100^{2}+2 \cdot 100 \cdot 80000 \cdot 0.042+80000^{2} \cdot 0.042^{2}\right]}=144.2 \mathrm{~cm}^{2} \\
& F_{32}=1 / 24 \sqrt{\left[100^{2}+2 \cdot 100 \cdot 80000 \cdot 0.038+80000^{2} \cdot 0.038^{2}+3 \cdot 100^{2} \cdot 1.986^{2}\right]}=131.6 \mathrm{~cm}^{2} \\
& F_{33}=1 / 24 \sqrt{\left.100^{2}+2 \cdot 100 \cdot 80000 \cdot 0+80000^{2} \cdot 0+3 \cdot 100^{2} \cdot 2.71^{2}\right]}=20 \mathrm{~cm}^{2}
\end{aligned}
$$

Let us take $h=70 \mathrm{~cm}$ cross-sectional height. Let us determine the forces by eccentricity. Here we have $N_{x}=100 \mathrm{kN} ; M_{x}=(300 \cdot 4-50 \cdot 4 \cdot 2)+100 \cdot 0.05=805 \mathrm{kN} \cdot \mathrm{m}=80500 \mathrm{kN} \cdot \mathrm{cm}$; $Q_{y}=300-200=100 \mathrm{kN}$.

$$
\begin{aligned}
& F_{31}=1 / 24 \sqrt{\left.100^{2}+2 \cdot 100 \cdot 80500 \cdot 0.043+80500^{2} \cdot 0.043^{2}\right]}=148.4 \mathrm{~cm}^{2} \\
& F_{32}=1 / 24 \sqrt{\left.100^{2}+2 \cdot 100 \cdot 80500 \cdot 0.0395+80500^{2} \cdot 0.0395^{2}+3 \cdot 100^{2} \cdot 1.976^{2}\right]}=137.4 \mathrm{~cm}^{2} \\
& F_{33}=1 / 24 \sqrt{\left.100^{2}+2 \cdot 100 \cdot 80500 \cdot 0+80500^{2} \cdot 0+3 \cdot 100^{2} \cdot 2.742^{2}\right]}=20.2 \mathrm{~cm}^{2}
\end{aligned}
$$

We take $h=60 \mathrm{~cm}$ and the area $F_{3}=144.2 \mathrm{~cm}^{2}$.

4) Let us analyze the cross-section at the distance of $6 \mathrm{~m}$ from its supporting structure (the middle of the span). Let us take $h=70 \mathrm{~cm}$ cross-sectional height. Let us take $h=70 \mathrm{~cm}$ height. Let us determine the forces by eccentricity. Here we have $N_{x}=100 \mathrm{kN} ; M_{x}=(300 \cdot 6-$ $50 \cdot 6 \cdot 3)+100 \cdot 0.05=905 \mathrm{kN} \cdot \mathrm{m}=90500 \mathrm{kN} \cdot \mathrm{cm} ; Q_{y}=300-300=0 \mathrm{kN}$. 


$$
\begin{aligned}
& F_{41}=1 / 24 \sqrt{\left[100^{2}+2 \cdot 100 \cdot 90500 \cdot 0.043+90500^{2} \cdot 0.043^{2}\right]}=162.62 \mathrm{~cm}^{2} \\
& F_{42}=1 / 24 \sqrt{100^{2}+2 \cdot 100 \cdot 90500 \cdot 0.0395+90500^{2} \cdot 0.0395^{2}+0}=153.1 \mathrm{~cm}^{2} \\
& F_{43}=1 / 24 \sqrt{\left[100^{2}+2 \cdot 100 \cdot 80500 \cdot 0+80500^{2} \cdot 0+0\right]}=4.2 \mathrm{~cm}^{2}
\end{aligned}
$$

Let us take $h=80 \mathrm{~cm}$ cross-sectional height. Let us determine the forces by eccentricity. $N_{x}=100 \mathrm{kN} ; M_{x}=(300 \cdot 4-50 \cdot 4 \cdot 2)+100 \cdot 0.1=910 \mathrm{kN} \cdot \mathrm{m}=91000 \mathrm{kN} \cdot \mathrm{cm} ; Q_{y}=0 \mathrm{kN}$.

$$
F_{4}=1 / 24 \sqrt{\left[100^{2}+2 \cdot 100 \cdot 91000 \cdot 0.041+91000^{2} \cdot 0.041^{2}+0\right]}=159.62 \mathrm{~cm}^{2}
$$

We take $h=80 \mathrm{~cm}$ and the area $F_{4}=59.62 \mathrm{~cm}^{2}$.

5) The girder integral capacity with account of the symmetry is:

$$
V=[(79.78+112.9) / 2 \cdot 200+(112.9+144.2) / 2 \cdot 200+(144.2+159.62) / 2 \cdot 200]=150720 \mathrm{~cm}^{3}
$$

The girder with uniform cross-section integral capacity is $V=(159.62 \cdot 600) \cdot 2=191544 \mathrm{~cm}^{3}$.

Theoretically we save $|191544-150720| / 191544 \cdot 100 \%=21.3 \%$ of steel.

\section{Conclusion}

In real life, the construction also requires the account of the cross-section flanges and walls local buckling resistance, bending limitations, limitations connected with width, depth and size unification of flanges and walls. Still if we take all these limitations into consideration, the whole effect of steel saving is about $10-20 \%$ compared with a girder or a beam with uniform cross-section.

\section{References}

1. Chang-Yu Cui, Bao-Shi Jiang, Engineering Structures 77, 109-118 (2014)

2. L.E. Mavromatidis, Energy and Buildings 106, 192-202 (2015)

3. I.I. Goldenblat, V.A. Kopnov, Strength and plasticity criteria of construction materials (Mashinostrojenie, Moscow, 1968)

4. Ya.I. Olkov, I.S. Kholopov, Steel pre-stressed trusses optimal design (Stroyizdat, Moscow, 1985)

5. I.S. Kholopov, Bar systems optimization as applied to SAE (computer-aided engineering systems), Doctoral dissertation (Moscow, 1972)

6. I.S. Kholopov, Mathematical modeling of bar systems volume by use of modular forms (textbook) (Kuibyshev State University ed., 1982)

7. Yu.A. Radtsig, Statically indeterminate trusses of minimum weight (Kazan University ed., 1969)

8. V.Yu. Alpatov, A.A. Lukin, Procedia Engineering 111, 20-29 (2015)

9. I.S. Kholopov, Building Mechanics and Structures Calculation, 14-17 (1981)

10. I.S. Kholopov, I.V. Loseva, Architecture and Civil engineering 5, (1987)

11. I.S. Kholopov, M.I. Balzannikov, A.O. Lukin, A.V. Solovjev, Vestnik of MGSU 11, 34-41 (2013)

12. I.S. Kholopov, I.V. Loseva, Higher education establishments news: Architecture and civil engineering 1, 54-58 (1980)

13. M.I. Balzannikov, B.G. Ivanov, A.A. Mikhasek, Vestnik MSSU, ScientificTechnological Journal 7, 119-124 (2012) 
14. N.V. Efimov, E.R. Rozendorn, Linear algebra and multidimensional geometry (Moscow, 1970) 